\title{
The theory of relativistic spontaneous emission from hydrogen atom in Schwarzschild Black hole
}

\author{
Jahangir A. Dar \\ Innovator at USIC Department, University of Kashmir, Srinagar, J\&K, India
}

Email address:

Jahangirahmad63@gmail.com

To cite this article:

Jahangir A. Dar. The Theory of Relativistic Spontaneous Emission from Hydrogen Atom in Schwarzschild Black Hole. American Journal of Astronomy and Astrophysics. Vol. 2, No. 6, 2014, pp. 66-71. doi: 10.11648/j.ajaa.20140206.12

\begin{abstract}
The mechanism of spontaneous emission radiated by the relativistic hydrogen atom falling radially towards Schwarzschild black hole, on the basis of Newtonian mechanics and Bohr's atomic theory is presented here. The energy radiated by this hydrogen atom is calculated as, $\zeta=A_{R}\left(m_{o} \tau / M_{B}\right)-m_{o} c^{2}$, where, $A_{R}$ is a constant. The relation for Lorentz factor $\gamma$ of relativity with mass $M_{B}$ of collapsed star and $m_{o}$ initial mass of particle is also derived. Furthermore, Hawking's energy relation for black holes has been derived also from the spontaneous energy relations using same boundary conditions the Hawking radiation possess. The frequency of energy spectrum has been found fall in gamma region of electromagnetic spectrum with range of $10^{23}$ and $10^{24} \mathrm{~Hz}$.
\end{abstract}

Keywords: Gravitational Radiation, Electromagnetic Radiation, Schwarzchild Black Hole, Hawking Radiation, Newtonian Mechanics, Bohr Atomic model and Special Relativity

\section{Introduction}

The gravitation radiation either in the collision process or fall of relativistic particles $[1,2,12,13,17]$ has been of considerable interest from last few centuries. It is believed also that electromagnetic radiation is possible if the charged particles like electron is moving in strong gravitational field $[1,4,5,6,7,12,13,17]$ like Schwarzschild black hole [2] or Kerr black hole [3], but the electromagnetic process is still in need to be studied more. To know more about the phenomenon of electromagnetic radiation from a charged particle moving in strong gravitational field, the works are done in Reff. No. 1-7 is advisable to read.

To calculate radiated energy from relativistic particle like Ruffini [6] and Cardos, Lenos and Yoshida[1] did, Lorentz factor or commonly called as gamma factor of relativity, $\gamma$, has been used with its different values like, $\gamma<<1$ by Ruffini [6] and $\gamma \leq 10$ by Cardos, Lenos and Yoshida[1]. For any particle with $\gamma \approx \infty$, it is much difficult to numerically obtain the total radiated energy emission [1]. It must be noted that for the total gravitational energy, $\gamma$ factor is treated same as in electromagnetic radiation energy. The relation for gravitational energy radiated is $\xi_{\mathrm{g}}=0.26 \mathrm{~m}^{2} \gamma^{2} / M_{B}$ and for electromagnetic energy radiated is $\xi_{\mathrm{r}}=\left(0.038 q^{2} / M_{B}\right) \gamma \log 2 \gamma$ (where $\gamma \rightarrow \infty$ ), here, $m$ is mass of particle, $M_{\mathrm{B}}$ is the mass of
Black hole, $q$ is the charge of the particle and $\gamma=\left(1-\beta^{2}\right)^{-1 / 2}, \beta$ $=v / c$ where $v$ is the velocity and $\mathrm{c}=3 \times 10^{-8} \mathrm{~ms}^{-1}$ is speed of light in vacuum. The beauty of this present paper is that the relation of $\gamma$ with other factors like mass of gravitational source $\mathrm{M}_{\mathrm{B}}$ and initial mass of particle itself is determined.

Some of physicists like me believe that even spontaneous emission is possible from the relativistic particles, in strong gravitational field but there is not any well defined process which explains the emission. The only slight idea about the spontaneous emission in Black-Hole-System is available in Hawking's radiation process [10,14], where it is argued that black hole emit thermal radiation via a quantum spontaneous emission [10] but it is not the property of particle as it does in electromagnetic or gravitational radiation emissions.

The main motive of this paper is to define the phenomenon of spontaneous emission by relativistic single electron atom like hydrogen in a strong gravitational field like Schwarzschild black hole. This indeed is the first attempt that has been ever taken in the science to define the process of spontaneous emission from such atom in any strong gravitational field by using combination of Newtonian mechanics, relativistic effects and Bohr's atomic model [23, 24]. Mean while, with spontaneous energy emission $\xi_{\mathrm{s}}$ by the 
atom the relation for its relativistic Lorentz factor $\gamma$, in any gravitational field with the mass of its source and the initial mass of electron $\mathrm{m}_{\mathrm{e}}$ of single electron atom has been also determined in the paper, also so that to determine the Hawking's energy relation, $\xi_{H}=\frac{\hbar c^{3}}{8 \pi G M_{B}}$.

\section{Formulation}

Consider any atom radially falling towards any nonrotating collapsed star like Schwarzhild black hole of mass $\mathrm{M}_{\mathrm{B}}$. During its radial fall its distance $x$ from the centre of collapsed star decreases and at any instant of time due to Newtonian mechanics $[11,15]$, the force of attraction, $F_{G}$, is given as;

$$
F_{G}=\frac{G M_{B} m}{x^{2}}
$$

where, $\mathrm{G}$ is the gravitational constant, $m$ is the mass of atom and $x$ is the distance between the gravitational centers of the two bodies. Taking the gravitational effect on the constituents of atom, i.e. electrons and nucleus independently, therefore, we have gravitational attractions for electron of mass $m_{e}$ and nucleus of mass $m_{n}$ as;

$$
\begin{aligned}
& F_{e}=\frac{G M_{B} m_{e}}{x^{2}} \\
& F_{n}=\frac{G M_{B} m_{n}}{x^{2}}
\end{aligned}
$$

The total attraction $F_{G}$ between the atom and the collapsed star is

$$
F_{G}=F_{e}+F_{n}
$$

Since the radial free fall of atom its velocity exceeds with decrease in $x$, therefore due to special relativity the mass of atom and thus of its constituents exceeds. This exceed in mass $[15,16]$ is given as;

$$
\mathrm{m}_{\mathrm{r}}=\mathrm{m}(1-\beta)^{-1 / 2}=\mathrm{m}_{\mathrm{r}} \gamma
$$

and for its constituents as,

$$
\begin{aligned}
& \mathrm{m}_{\mathrm{e}^{*}}=\mathrm{m}_{\mathrm{e}}(1-\beta)^{-1 / 2}=\mathrm{m}_{\mathrm{e}} \gamma \\
& \mathrm{m}_{\mathrm{r}^{*}}=\mathrm{m}_{\mathrm{n}}(1-\beta)^{-1 / 2}=\mathrm{m}_{\mathrm{n}} \gamma
\end{aligned}
$$

Now, consider the hydrogen atom, ${ }^{1} H$, consisting of an electron, $\mathrm{e}^{-}$, and proton, $\mathrm{p}$, falling radially towards a Schwarzchild black hole. Both $\mathrm{e}^{-}$and $\mathrm{p}$ will possess gravitational attraction with the Schwarzschild black hole of mass $M_{B}$. Total gravitational force will be given by the eq. (1). Relativistic mass of its constituents is given by eq. (6) and (7).
In Bohr model [23, 24] of ${ }^{1} H$ atom, the radius occupied by an electron revolving around the nucleus is given as;

$$
a_{o}=\frac{4 \pi \varepsilon_{o} \hbar^{2} n^{2}}{m_{e} e^{2}}
$$

When relativistic effects are taken

$$
a_{r}=\frac{4 \pi \varepsilon_{o} \hbar^{2} n^{2}}{m_{e} \gamma e^{2}}
$$

Relativistic conditions require $a_{r}<a_{o}$, since $m_{e} \gamma>m_{e}$. Thus there is contraction in ${ }^{1} H$ atom during its free fall towards any strong gravitational star. Such contraction effect has been studied for many atoms like $\mathrm{Hg}, \mathrm{Ag}$, etc, see ref. no. $[18,19]$. However, there the effect is due to the orbital motion of $e^{-}$around the nucleus $[18,19]$. If the decrease in orbital radius $a_{o}$ is quite high, as possible if the atom fall towards strong gravitational field as in our ${ }^{1} H$, it will led to the transaction of electron from one energy level to another. This transaction of electron from higher energy state to lower energy state led to the release of 'spontaneous emission' from the ${ }^{1} H$ atom.

The amount of energy released in the form of spontaneous emission is equal to the difference between energies possessed by electron at its initial energy level, when $x \rightarrow \infty$, and final energy level at $R_{S} \leq x \triangleleft \infty$, i.e.

$$
\xi=\xi_{\mathrm{f}}-\xi_{\mathrm{i}}
$$

Using Bohr's model of atom [23, 24], we have, energy $\xi_{\mathrm{o}}$ occupied by electron at any energy level $n$ as;

$$
\xi_{\mathrm{o}}=\frac{m_{e} e^{4}}{32 \pi^{2} \varepsilon_{o}^{2} \hbar^{2} n^{2}}
$$

in eq. (11), $\varepsilon_{o}$ is permittivity of free space, $n$ is the energy level also called as principal quantum no. in quantum mechanics, $\hbar$ is reduced Planck's constant and $\pi$ is 3.14 (approx.).

Therefore, eq. (10) transforms as;

$$
\xi=\frac{m_{e}(\gamma-1) e^{4}}{32 \pi^{2} \varepsilon_{o}^{2} \hbar^{2}\left(n_{1}^{2}-n_{2}^{2}\right)}
$$

where, $\lambda m_{o}$ is the mass possessed by $\mathrm{e}^{-}$at its new energy level $\mathrm{n}_{\mathrm{f}}$ at $R_{S} \leq x \triangleleft \infty$ and $m_{o}$ mass at initial energy state $n_{i}$ at $x \rightarrow \infty$

By Einstein's energy-mass equivalence relation, $\xi=\Delta \mathrm{mc}^{2}[20,21]$. Using this equivalence relation and inserting the eq. (11) in eq. (10), the transformed relation comes

$$
\xi=\left(\gamma \mathrm{m}_{\mathrm{e}}-\mathrm{m}_{\mathrm{e}}\right) \mathrm{c}^{2}=\mathrm{m}_{\mathrm{e}}(\gamma-1) \mathrm{c}^{2}
$$


where, $\Delta \mathrm{m}$ is the total change in mass of $e^{-}$by the relativistic effects, equal to the difference between the relativistic mass $2 \mathrm{~m}_{\mathrm{e}}$ and initial mass $m_{e}$ of $\mathrm{e}^{-}$. The relativistic effective mass of proton has been neglected since the radiation can be assumed only by the fall of electron from one energy level to other, and total change in distance between the centers of two particles is same to the change in distance of $e^{-}$from the centre of $\mathrm{p}^{+}$.

\section{Deriving Value of Lorentz Factor}

As stated earlier in all calculations related to energy emission by relativistic particle in strong gravitational field, the value of Lorentz factor [22] is assumed. Here we will derive it relation with the strength of collapsed star.

Due to the influence of of gravitational force on radially falling ${ }^{1} H$, its momentum changes with time $\tau$ of free fall, and thus momentum of $e^{-}$changes. Taking $\gamma \mathrm{m}_{\mathrm{e}} \mathrm{v}$, as final momentum, say somewhere near event horizon and $\mathrm{m}_{\mathrm{e}} \mathrm{v}_{\mathrm{o}}$ as initial momentum somewhere at infinity and $\tau$ as time taken for total momentum change by $e^{-}$, then Newtonian mechanics [11] says,

$$
\mathrm{F}_{\mathrm{m}}=\frac{\gamma \mathrm{m}_{\mathrm{e}} \mathrm{v}-\mathrm{m}_{\mathrm{e}} \mathrm{v}_{\mathrm{o}}}{\tau}=\frac{\not \mathrm{m}_{\mathrm{e}} \mathrm{v}}{\tau}
$$

The value of initial momentum $m_{o} v_{o}$ has been neglected by assuming the atom was initially at rest or it was moving towards another direction other than the direction of its radial fall.

In the mean time electron as it satisfies eq. (14), it must also satisfy the gravitational relation in eq. (2), i.e. $F_{G}=F_{m}$. After some transformations the condition derives the relation for Lorentz factor as;

$$
\gamma=\frac{G M_{B} \tau}{x^{2} v}
$$

Any information about any particle falling towards black hole can be obtained as long as it resides beyond event horizon of radius $R_{S}$, what happens to particle after it crosses it no physics is known to define it, therefore, the value of Lorentz factor can go maximum at $x=R_{S}$. Where $R_{S}$ is Schwarzschild's radius, given as $\mathrm{R}_{\mathrm{S}}=2 \mathrm{GM}_{\mathrm{B}} / \mathrm{c}^{2}$. Interchanging $x$ by $R_{S}$ in eq. (15), we arrive on the result

$$
\gamma=\frac{\mathrm{c}^{4} \tau}{4 \mathrm{GM}_{\mathrm{B}} \mathrm{V}}
$$

What the smart conclusions we have in eq. (15-16)! For any radially falling particle towards the collapsed star, Lorentz factor depends also on $\tau$ and $M_{B}$, not only on velocity $v$ as we know so far.

\section{Transformation Spontaneous Energy Emission Relations}

Eq. (15) and (16), has led us to new values of Lorentz factor $\gamma$, these new values of $\gamma$ can be implanted into our previously derived relations for spontaneous emissions, as eq. (12 \& 13), of ${ }^{1} \mathrm{H}$ during its radial free fall due to the Schwarzschild black hole. The transformed relations are;

$$
\begin{gathered}
\xi=\frac{m_{o}\left(\frac{G M_{B} \tau}{x^{2} v}-1\right) e^{4}}{32 \pi^{2} \varepsilon_{o}^{2} \hbar^{2}\left(n_{1}^{2}-n_{2}^{2}\right)}, \text { at } R_{S}<x<<\infty \\
\xi=\frac{m_{o}\left(\frac{c^{4} \tau}{4 G M_{B} v}-1\right) e^{4}}{32 \pi^{2} \varepsilon_{o}^{2} \hbar^{2}\left(n_{1}^{2}-n_{2}^{2}\right)}, \text { at } R_{S} \cong x \\
\xi=m_{o}\left(\frac{G M_{B} \tau}{x^{2} v}-1\right) c^{2} \text { at } R_{S}<x<<\infty \\
\xi=m_{o}\left(\frac{c^{4} \tau}{4 G M_{B} v}-1\right) c^{2} \text { at } R_{S} \cong x
\end{gathered}
$$

Eq. (17 \& 18) give the relation for spontaneous energy emission by ${ }^{1} H$ during its radial fall towards Schwarzschild black hole, in terms of Bohr's atomic model at conditions when it is near to event horizon or far from it, while at the same two conditions, Eq. (19 \& 20) give the relation for spontaneous emission in terms of Einstein's mass-energy equivalence relation $[20,21]$.

\section{Derivation of Hawking's Black Hole Energy Emission Relation}

Stephen Hawking suggested that black holes possess temperature $[14,25]$ well known as Hawking temperature, $T_{H}=\hbar c^{3} / 8 \pi G M_{B} K_{B}$, where $k_{B}$ is Boltzmann's constant, therefore, emit energy [14, 25],

$$
\xi_{H}=k_{B} T_{H}=\frac{\hbar c^{3}}{8 \pi G M_{B}}
$$

The only possibility of the formation of Hawking radiation is at event horizon, that is, for Hawking radiation it must satisfy the condition, $\xi_{H}=\xi_{H}\left(R_{S}\right)$. Our relations $18 \& 20$, for spontaneous energy emission due to relativistic atom also satisfies the same condition, $\xi=\xi\left(x \cong R_{S}\right)$. Now, consider in eq. (20), $v \rightarrow c$ and $\tau$ as some constant and initial energy $m_{o} c^{2}=0$, therefore, the said relation gets transformed as;

$$
\xi=m_{o}\left(\frac{c^{3}}{4 G M_{B}}\right) c^{2} \tau=m_{o}\left(\frac{\hbar c^{3}}{8 \pi G M_{B}}\right) j
$$


Where, $j=2 \pi c^{2} \tau / \hbar$ is a constant and $\tau$ is taken as any known quantity.

It is clear from eq. (22), that the Hawking energy emission is a simple case of our spontaneous emission when the mass $m_{o}$ is neglected. Elsewhere, Hawking's energy emission is the property of the mass $M_{B}$ of black hole only, it doesn't define the fact that by quantum mechanics as all types of pair particles like electron-neutrino, tau-antitau, etc, can be created by vacuum due to strong gravitational field as per Hawking process then what is the effect on radiated energy due to the fall of these different particles possessing different properties is not defined and this indeed is one of the greatest drawbacks of Hawking's radiation process. Now look at our eq. (22), it defines clearly the effect on radiation emission due to the fall of different particles with different properties like mass towards black hole. However, in Hawking process the black hole losses some of its mass [14, 25], but in our spontaneous emission it is not defined and has been made mysterious here. In my upcoming work am going to explain and solve the mystery.

To derive Hawking radiation we have neglected some

values of eq. [20] and reduced it to Hawking's relation, however the actual relation can be transformed and reduced to simpler relation as;

$$
\xi=A_{R} \frac{m_{o} \tau}{M_{B}}-m_{o} c^{2} \text { at } x=R_{S}, v=c
$$

where, $A_{R}$ is a constant of value $=1.4503 \times 10^{51} \mathrm{~L}^{3} \mathrm{Kg}^{2} \mathrm{~s}^{-5} \mathrm{~N}^{-1}$. Now look at our eq. (23), it defines clearly the effect on radiation emission due to the fall of different particles having different rest masses towards black hole, mass of black hole, time $\tau$ and the initial energy of particle. Clearly, the radiation due to the fall of particle moving with the speed of light at event horizon depends on functions $m_{o}, M_{B}$ and $\tau$, that is, $\xi=\xi\left(m_{o}, M_{B}, \tau\right)$.

\section{Numerical Results}

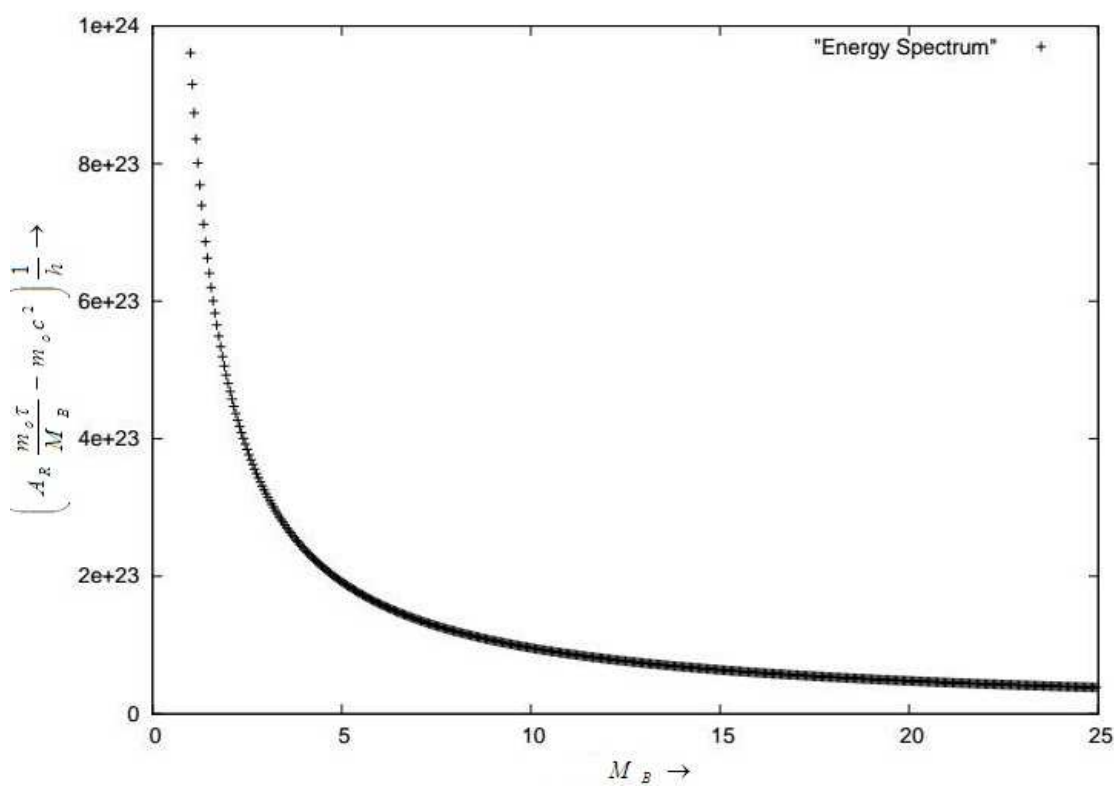

Fig. 1. The electromagnetic spectrum for the hydrogen atom falling into Schwarzschild black hole at $x=R_{S}$ and by considering $\tau=1$.

In order to obtain numerical results as well band spectrum radiated by single electron hydrogen atom during its radial fall in Schwarzschild black hole, in terms of frequency, eq. (23) is considered, that is, when radiated energy follows the condition $\mathrm{x}=$ Rs. For calculating the results, the value $\tau=1$ and the mass $\mathrm{mo}=9.1 \times 10^{-31} \mathrm{Kg}$ as the standard value of electron has been taken. Fig. 1 shows the frequency $v$ range of this one electron $\mathrm{H}$ atom when it fall into Schwarzschild black hole of mass taken in solar masses $M_{O}$.In deriving the energy spectrum Planck's constant $h$ has been also introduced for relation $v=\xi / h$. The energy spectrum from the calculations is calculated to fall in gamma region of electromagnetic spectrum of range $10^{23} \mathrm{~Hz}$ and $10^{24} \mathrm{~Hz}$. In Fig. 2, a typical result for the value of Lorentz factor $\gamma$ is shown, by considering the same as conditions as used for energy spectrum. The equation no. 16 has been considered for results. Here one more assumption has been taken, that is, $v \cong c$. It is obvious from the two figures that the slope of results is similar, this means the energy spectrum depend on the $\gamma$ factor only and is independent on the initial energy $m_{o} c^{2}$ of the falling hydrogen atom into the Schwarzschild black hole. Therefore the term $m_{o} c^{2}$ can be neglected from the equation 23 and thus the equation can be simply written as;

$$
\xi=A_{R} \frac{m_{o} \tau}{M_{B}} \text { at } x=R_{S}, v=c
$$


If $m_{o} c^{2}$ could have been greater than the term $A_{R} \frac{m_{o} \tau}{M_{B}}$, diverge towards the straight line, where it is not. Therefore the initial energy term can be neglected in the Eq. (23). that is, $m_{o} c^{2}>A_{R} \frac{m_{o} \tau}{M_{B}}$, then the slope in FIG. 1 should

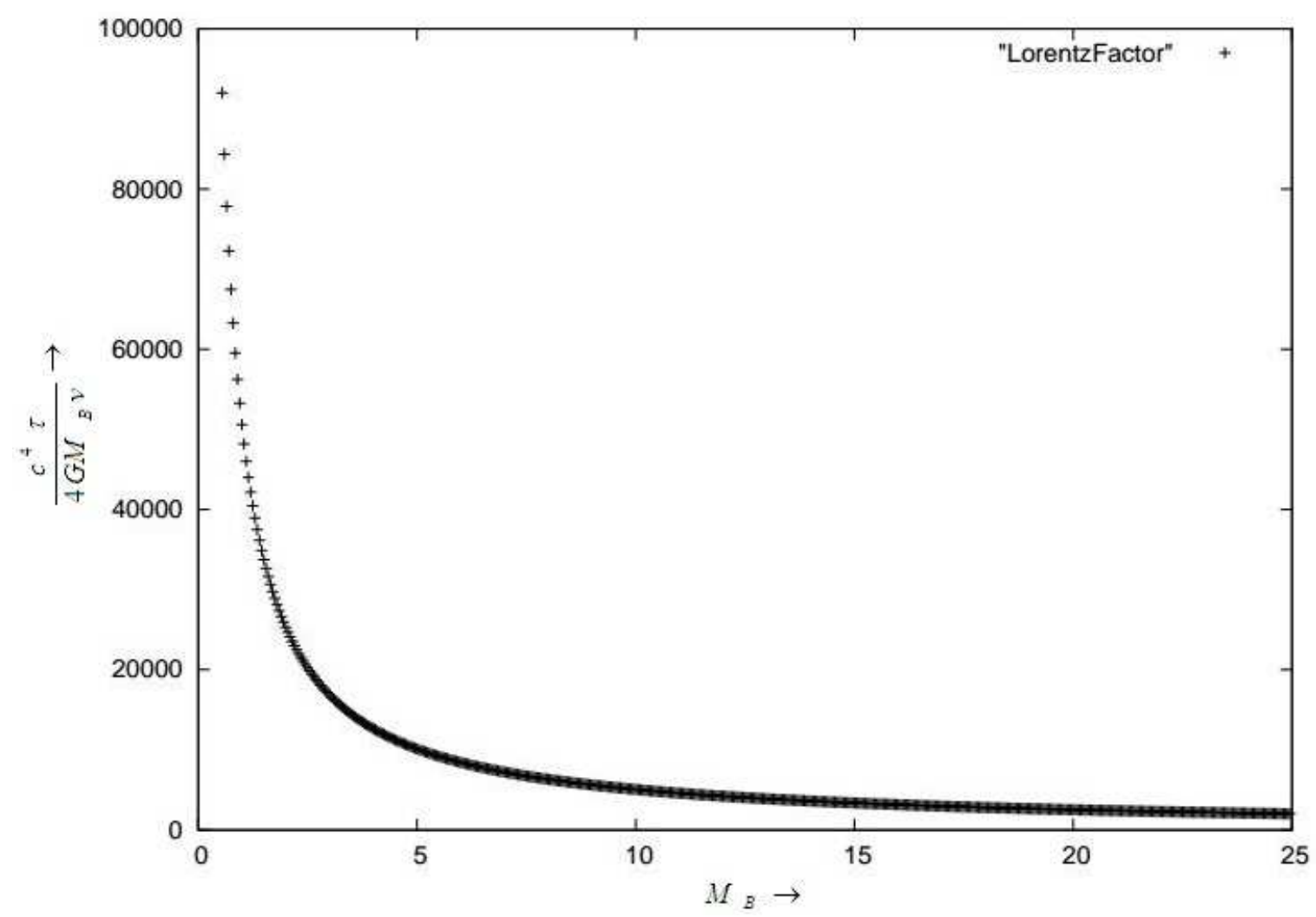

Fig. 2. Variation of Lorentz factor $\lambda$ for the hydrogen atom falling into Schwarzschild black hole at $x=R_{S}$ and by considering $\tau=1$.

\section{Conclusion}

The spontaneous energy emission, has been derived on the basis of Newtonian and Relativistic mechanics with the help of Bohr's Atomic Model. Energy relations as equations [17, $18,19,20,23]$ has been derived using these classical ideas, at different conditions. It is found in some regards, the energy emission is similar as have been predicted by Hawking's arguments. The only difference between Hawking's and this classical relativistic radiation emission from black hole system is in the former energy emission is independent on the property of falling particle and in the later the radiation emission is dependent on the initial mass as well on initial energy of the falling particle, as obvious from eq. [21, 23].

\section{Acknowledgements}

I performed this work without the support of any grant from any private or govt. institution/agency. I am thankful to my college Prof. Dr. Shashikanth Gupta at Amity University Haryana for providing me the required information regarding plotting of graphs, and am deeply thankful to my family members for providing me all required financial support and especially during my stay at Amity University Haryana, India.

\section{References}

[1] V. Cardoso, J. P. S. Lemos, S. Yoshida, Phys. Rev. D 68, 084011 (2003).

[2] V. Cardoso, J. P. S. Lemos, Phys. Lett. B 538, 1 (2002).

[3] V. Cardoso, J. P. S. Lemos, Gen. Rel. Gravitation 35, 327-333 (2003); Phys. Rev. D 67, 084005 (2003).

[4] S. Chandrasekhar, S. Detweiler, Proc. R. Soc. London A 344, 441 (1975); S. Chandrasekhar, The Theory of Black Mathematical Holes, (Oxford University Press, New York, 1983).

[5] M. Johnston, R. Ruffini, F. Zerilli, Phys. Lett. 49B, 185 (1974).

[6] R. Rufini, Phys. Lett. 41B, 334 (1972).

[7] F. Zerilli, Phys. Rev. D2, 2141 (1970); Phys. Rev. D9, 860 (1974).

[8] Hawking, S. W., Commun. Math. Phys. 43, 199-220(1975) 
[9] Jacob D. Bekenstein, Phys. Rev. D 9, 3292(1974)

[10] S. Weinfurtner, E. W. Tedford, M. C.J. Penrice, W. G. Unruh, G. A. Lawrence, Phys. Lett. 106, 021302 (2011).

[11] S. N. Gupta, Rev. Mod. Phys. 29, 334 (1957).

[12] R. Ruffini, M. Sasaki, Prog. of Theor. Phy. 66, 5 (1981).

[13] D. G. Yakovlev, Zh. Esp. Teor. Fiz. 68, 369-376 (1975).

[14] Hawking, S. W., Nature, 248, 30-31 (1974).

[15] L. B. Okun, Usp. Fiz. Nauk 158, 511—530 (1989).

[16] C. G. Alder, A. J. Phys. 55, 8 (1987).

[17] M. Davis, R. Ruffini, Phys. Rev. Lett., 27, 21 (1971).
[18] P. Pyyko, J. P. Desclaux, Acc. Chem. Res., 12 (1979).

[19] S. J. Rose, I. P. Grant, N. Pyper, J. Phys. B: At. Mol. Phys., 11, 1171 (1978).

[20] A. Einstein, Annalen der Physik 18, 13 (1905).

[21] L. B. Okun, Phys. Today, 42, 6 (1969).

[22] J. R. Forshaw, A. G. Smith, "Dynamics and Relativity", Wiley, 2009.

[23] N. Bohr, Philosophical Magazine, 26, 151 (1913).

[24] N. Bohr, Nature, 107, 2682 (1921).

[25] Hawking, S. W., Phys. Rev. D 13, 191 (1976). 